\title{
Distributed Raman Amplification for Long-Haul Optical Frequency Dissemination
}

\author{
C. Clivati*, D. Calonico, F. Levi, A. Mura \\ Istituto Nazionale di Ricerca Metrologica \\ strada delle cacce 91, Torino, Italy \\ *also with Politecnico di Torino, \\ corso Duca degli Abruzzi,Torino, Italy

\section{G. Bolognini} \\ IMM Institute, National Research Council CNR \\ via Gobetti 101, Bologna, Italy
}

\author{
S. Faralli, \\ Istituto TECIP, Scuola Superiore Sant'Anna \\ via G. Moruzzi 1, Pisa, Italy
}

N. Poli

Dip. Fisica e Astronomia and LENS, Università di Firenze and Istituto Nazionale di Fisica Nucleare, sez. Firenze via Sansone 1, Sesto Fiorentino (FI), Italy

\begin{abstract}
This work describes the use of Distributed optical Raman Amplification for the realization of coherent optical fiber links for frequency dissemination. The main advantages of this technique are high gain and feasibility of long fiber spans with a simple apparatus, without degrading the link stability.
\end{abstract}

Keywords-fiber links, optical amplifiers, Raman amplification

\section{INTRODUCTION}

In recent years, phase-compensated optical links proved to be the most performing technique to disseminate optical frequencies on a continental scale [1,2]. Most National Metrology Institutes are thus cooperating to realize a panEuropean fiber network, that will enable optical clocks comparisons at unprecedented levels of accuracy and stability. The ultrastable frequency transfer via optical fiber requires that the fiber phase noise is compensated. This is done by reflecting a portion of the transferred light from the user end back to the transmitter end, and by comparing it to the original light. The double pass allows to cancel the phase noise accumulated along the fiber, provided that the optical path is exactly the same in both directions.

Dedicated amplification systems are thus needed, that must be fully bidirectional and symmetrical in the two directions. Bidirectional Erbium Doped Fiber Amplifiers are typically used, but their gain is limited by the back-reflections along the path, that may lead the amplifier to oscillate and may limit the achievable gain to about $20 \mathrm{~dB}$. Distributed amplification is quite promising from this point of view, and offers several advantages compared to BEDFA. Distributed Brillouin Amplifiers (DBA) have recently been demonstrated and fruitfully used in coherent optical links [3]. We demonstrate that Distributed Raman Amplification (DRA) is a reliable technique as well: it does not degrade the transfer of the optical carrier at the $10^{-19}$ level of stability, and allows to bridge hauls of up to $60 \mathrm{~dB}$ losses without intermediate stations [4].

Raman amplification is based on the power transfer between two optical beams, the pump and the signal, through stimulated scattering of light with optical phonons [5]. Since the power transfer is distributed along the fiber, with an effective length of about $20 \mathrm{~km}$, DRA can provide a very high gain without the onset of lasing or oscillations, even for high pump powers [6]. The gain is maximum when the signal is downshifted $\sim 13.2 \mathrm{THz}$ from the pump light, and the bandwidth is several $\mathrm{THz}$ wide around the gain peak. DRA requires pump power levels on the order of $\sim 1 \mathrm{~W}$, that is rather high, especially if compared to those required in DBA $(\sim 10 \mathrm{~mW})$. On the other hand, DRA is intrinsically bidirectional, i.e. it amplifies signals that propagate in the opposite or in the same direction as the pump (hereafter indicated as counter- and co-pumped), whilst DBA only amplifies counter-propagating signals. Also, thanks to the large gain bandwidth, DRA does not require a frequency control of the pump laser to ensure a stable gain. Thus, DRA technology could help to simplify long haul optical links and to increase the reliability of the whole infrastructure.

In this work, we propose an analysis of the gain, the optical signal to noise ratio (OSNR) and the phase noise of the amplified optical carrier, and report on the use of DRA in a coherent optical link based on $200 \mathrm{~km}$ of fiber spools, both in a counter-pumped and in a co-pumped scheme.

\section{THE EXPERIMENT}

\section{A. Optical Instrumentation and Experimental Apparatus}

The laboratory test-bed we used is sketched in Fig. 1. An ultrastable laser radiation at $1542 \mathrm{~nm}$ is generated by locking a fiber laser through the Pound-Drever-Hall technique to a Fabry-Perot high finesse $(120,000)$ cavity made of Corning Ultra Low Expansion (ULE) glass. The resulting laser linewidth is smaller than $30 \mathrm{~Hz}$ (details in [7]), with OSNR $56 \mathrm{~dB}$ in $0.1 \mathrm{~nm}$ resolution bandwidth. Light is coupled into a standard SMF-28 single mode fiber, with a total length of up to $200 \mathrm{~km}$. The light power at fiber input is $0.5 \mathrm{~mW}$; total losses due to fiber and connectors amount to $\sim 45 \mathrm{~dB}$. To 
achieve phase noise cancellation, two acousto-optic modulators (AOM) are employed. AOM1 is placed before the fiber link and used as actuator to compensate the fiber phase noise. AOM2 is placed after the fiber link to shift the back-reflected radiation and allows to distinguish the real signal from stray reflections. Photodiode PD1 detects the fiber noise from an interferometric scheme [7] and a phase-locked-loop (PLL) feeds AOM1 with the correction signal. The bandwidth of the PLL loop filter is $20 \mathrm{kHz}$, and a minimum round-trip optical signal power of at least $-70 \mathrm{dBm}$ has to combine to the original light on PD1 to phase-lock the optical carrier. Photodiode PD2 is used to compare the phase noise of the radiation transferred through the fiber to that injected at the input.

We tested both co-and counter-pumped DRA, by coupling the pump light into the fiber trough wavelength-division multiplexers WDM1 or WDM2 respectively. Two different pump lasers at $1450 \mathrm{~nm}$ were used. The first is a depolarized fiber Raman laser (FRL) delivering up to $\sim 800 \mathrm{~mW}$ into the fiber (OSNR $>50 \mathrm{~dB}$ in $0.1 \mathrm{~nm}$ resolution bandwidth). The other Fabry-Pérot laser (FPL) depolarized pump is composed of two polarization-multiplexed Fabry-Perot diode lasers at the same wavelength, providing $\sim 260 \mathrm{~mW}$ (OSNR $\sim 58 \mathrm{~dB}$ in 0.1 $\mathrm{nm}$ resolution bandwidth) at the fiber input [8]. The depolarization of the pump laser is mandatory, as the Raman gain is maximum for co-polarized pump and signal, whilst it is minimum when pump and signal are orthogonally polarized. Therefore, care must be devoted to minimize gain fluctuations associated to polarization mode dispersion in the fiber.

Also, to avoid the transfer of Relative Intensity Noise (RIN) from pump to signal, we used the higher-RIN $(\sim-110 \mathrm{~dB} / \mathrm{Hz})$ FRL pump at frequencies lower than $10 \mathrm{MHz}$ ) for counterpumping, and the lower-RIN ( - $140 \mathrm{~dB} / \mathrm{Hz})$ FPL for copumping. Indeed, counter-pumped DRA is known to be less affected by pump-to-signal RIN transfer than co-pumped DRA [9], since the RIN transfer is averaged out by the large difference in the group velocities of counter-propagating pump and signal. Being the signal RIN level <-141 dB/Hz, Raman amplification leads to an expected added signal RIN of $\sim-134.7 \mathrm{~dB} / \mathrm{Hz}$ in co-pumping, and $\sim-95.3 \mathrm{~dB} / \mathrm{Hz}$ in counterpumping, whose cross-talk values are extremely low when integrated over the narrow signal bandwidth $(30 \mathrm{~Hz})$.

\section{B. Measurements and Discussion}

We initially evaluated the DRA impact with a counterpump scheme. On-off gain, OSNR and the phase noise of the amplified radiation have been measured by performing on PD2 the heterodyne beatnote between the signal travelling along the link and the light from the short reference arm. Fig. 2 (circles) shows the measured gain after $200 \mathrm{~km}$ propagation, versus the fiber-coupled pump power. The gain increases with pump power and attains the maximum value of $23 \mathrm{~dB}$, limited by the effective power available with the FRL $(800 \mathrm{~mW})$. The measured OSNR is $106 \mathrm{~dB} / \mathrm{Hz}$, and is limited by the amplified spontaneous emission at pump powers higher than $600 \mathrm{~mW}$, whilst it is degraded at lower pump powers due to the electrical noise of PD2.

This work has been partly funded by Compagnia di San Paolo, by the Italian Ministry of Research, contract PRIN09, and by Ente Cassa di Risparmio di Firenze.

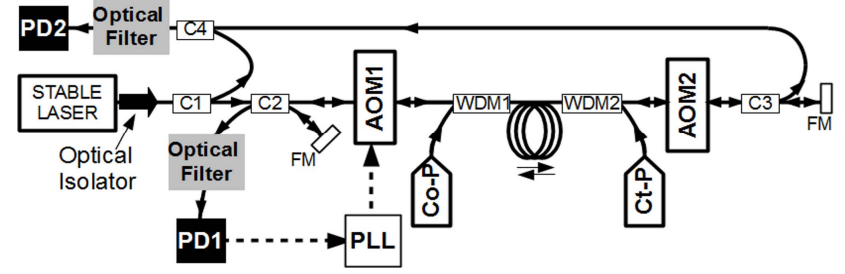

Fig. 1. Set up of the coherent fiber link with DRA. PD Photodiodes, C Couplers, FM Faraday Mirrors, WDM Wavelength Division Multiplexers, CoP Co-propagating Pump, Ct-P Counter-propagating Pump, AOM AcoustoOptic Modulators, PLL Phase Locked Loop.

To investigate the maximum link length achievable without intermediate stations, the FPL as well was coupled into the fiber as a co-pump. The overall gain then increased to $32 \mathrm{~dB}$, equivalent to a single stage DRA with a $1 \mathrm{~W}$ single counter-pump [8], limited by the power available with our FPL. The double stage pump allows to phase-compensate a link with up to $60 \mathrm{~dB}$ losses in a single span with our setup.

To test the flexibility of the amplifiers chain, we tried the high-power FRL as co-pump and the low-power FPL as counter-pump. We measured the co-propagating signal gain, as a function of the pump power while keeping a constant counter-pump gain of $9 \mathrm{~dB}$, and with an input signal of $\sim 0.5 \mathrm{~mW}$. At high co-pump power levels, we observed degrading effects on the signal, and a strong gain reduction, as shown in Fig. 2 (squares). This is expected to be due to the power level of the amplified signal, that increases beyond the SBS threshold in the co-pumped scheme. When the gain reaches $\sim 13 \mathrm{~dB}$, the amplified signal achieves $10 \mathrm{~mW}$, i.e. the SBS threshold. The net gain is then reduced by almost $10 \mathrm{~dB}$ with respect to the counter-pump scheme. This did not happen in the previous scheme, in which the signal amplified by the counter-pumped FRL was much weaker due to the fiber losses. As a result, in that scheme, the amplified signal never exceeded the SBS threshold [5]. Such detrimental effects can be reduced by a careful design of the amplifier chain whenever intermediate stations are required along the path.

Figure 3 shows the phase noise power spectral density $S_{\phi}(f)$ of a $100 \mathrm{~km}$ compensated link without (dashed grey line) and

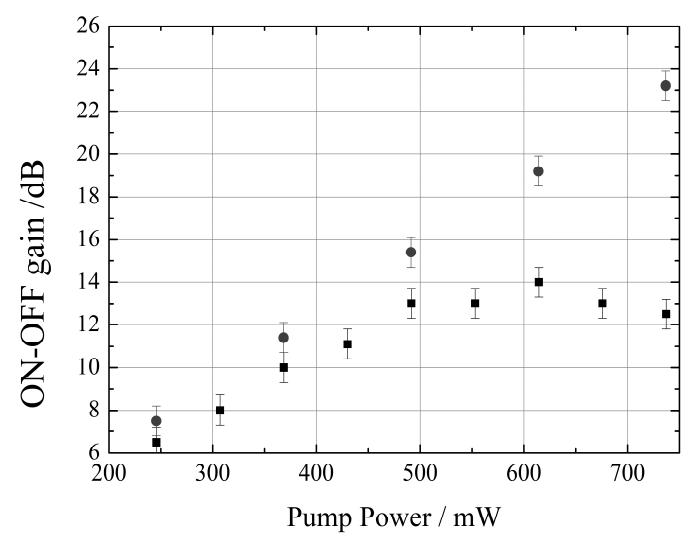

Fig. 2. Raman gain versus pump power in an optimized counter-pumped scheme (circles) and in a not optimized co-pumped scheme (squares). 


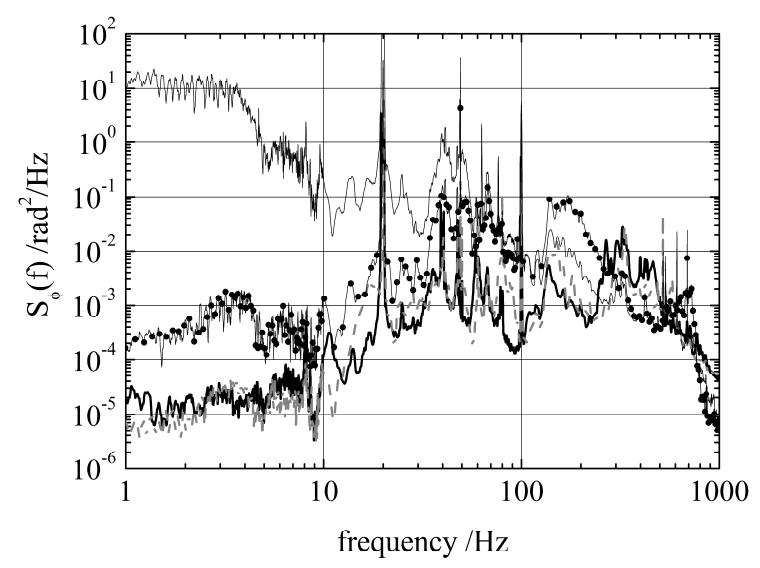

Fig. 3. Phase noise density $S_{\phi}(f)$ of the free running (thin black line) and compensated link (black line, filled circles) at $200 \mathrm{~km}$, and $S_{\phi}(\mathrm{f})$ of a $100 \mathrm{~km}$ link without amplification (dashed grey line) and with an amplification of 22 $\mathrm{dB}$ (thick black line).

with (solid black line) an optical amplification of $22 \mathrm{~dB}$. The spikes observed at frequencies within the link servo bandwidth $(500 \mathrm{~Hz})$ are mainly due to acoustic noise on the spooled fiber. The residual noise of the compensated link agrees with the expected limit due to the fiber delay [10], and there is no evidence of phase noise degradation due to DRA. Fig. 3 also shows the phase noise power spectral density of the freerunning link at $200 \mathrm{~km}$ (thin black line) and of the compensated link (black line, filled circles). Again, the noise of the $200 \mathrm{~km}$ agreed with the expectations, considering the phase noise of the free running link and the fiber delay [10].

Link stability over long averaging times was calculated by counting the beatnote on PD2 with a high resolution $\Lambda$-type counter [11]. The data frequency instability $\sigma_{\Lambda}(\tau)$ obtained by processing data from $\Lambda$-counters can be converted into the Modified Allan deviation for common phase noises [11]. Fig. 4 shows $\sigma_{\Lambda}(\tau)$ of the link at $100 \mathrm{~km}$ (circles), at $200 \mathrm{~km}$ (squares) and of the interferometer without fiber spools (diamonds, grey line). Up to $20 \mathrm{~s}$ the link noise is dominant,

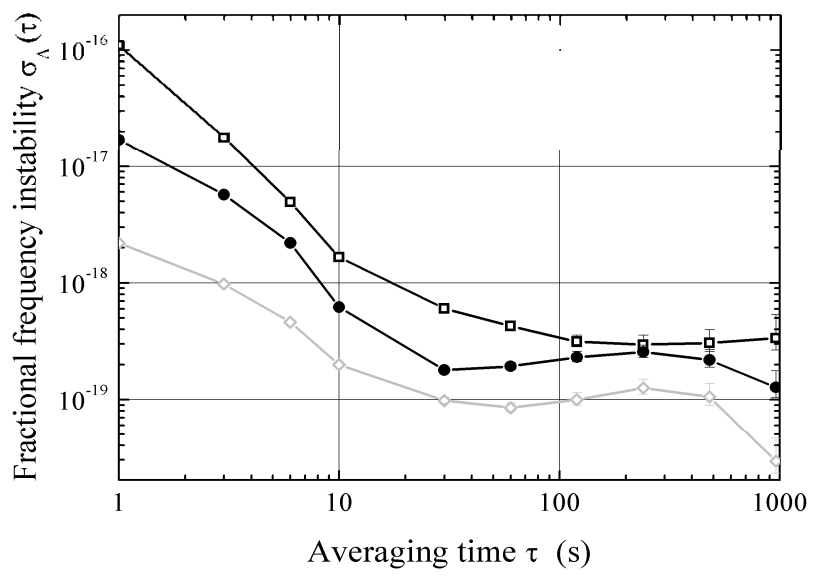

Fig. 4. Fiber link frequency instability on a $100 \mathrm{~km}$ link(filled circles), on the $200 \mathrm{~km}$ link using ROA (empty squares), and the interferometer noise floor (grey line, empty diamonds). whilst at longer times the stability reaches a floor of $\sigma_{\Lambda}(\tau) \sim 3 \cdot 10^{-19}$. We attribute this limit to random, not reciprocal phase variations related to the fiber birefringence. Nevertheless, these results show that DRA enables coherent amplification in phase-compensated optical links.

\section{CONCLUSIONS AND OUTLOOKS}

We demonstrate that DRA is suitable for amplification in phase coherent optical links, allowing to bridge hauls of $200 \mathrm{~km}$ with a single fiber span, with a frequency instability of $3 \cdot 10^{-19}$ at $100-1000 \mathrm{~s}$. DRA offers several advantages in terms of link maintenance and reliability with respect to discrete amplification techniques, providing a high gain with a simple apparatus. However, care should be devoted to the design of links involving both counter- and co-pumping, to avoid nonlinearities affecting the ultimate performances of the amplifier. We plan to investigate the use of DRA on a $650 \mathrm{~km}$ optical link that is under development_between INRIM in Turin and LENS-UNIFI in Firenze, Italy [12]. DRA could improve the present design, reducing the number of amplifiers, as nine BEDFA amplifiers are used at the moment.

\section{REFERENCES}

[1] K. Predehl, G. Grosche, S. M. F. Raupach, S. Droste, O. Terra, J. Alnis, et al., "A 920-Kilometer Optical Fiber Link for Frequency Metrology at the 19th decimal place," Science vol. 336, pp. 441-444, April 2012.

[2] O. Lopez, A. Haboucha, F. Kefelian, H. Jiang, B. Chanteau, V. Roncin, et al., "Cascaded multiplexed optical link on a telecommunication network for frequency dissemination," Opt. Expr. vol. 18, pp. 1684916857, August 2010.

[3] O. Terra, G. Grosche, and H. Schnatz, "Brillouin amplification in phase coherent transfer of optical frequencies over $480 \mathrm{~km}$ fiber," Opt. Expr. vol. 18, 16102-16110, July 2010.

[4] C. Clivati, G. Bolognini, D. Calonico, S. Faralli, F. Levi, A. Mura, N. Poli, "Distributed Raman optical amplification in phase coherent transfer of optical frequencies," IEEE Photonics Technol. Lett., in press.

[5] G. Agrawal, Nonlinear Fiber Optics, 3rd ed. San Diego: Academic Press, 2001 .

[6] M. Islam, "Raman Amplifiers for Telecommunications" IEEE J. Sel. Top. Quantum Elect. , vol. 8, pp. 548-559, May/Jun. 2002.

[7] C. Clivati, D. Calonico, C. E. Calosso, G. A. Costanzo, F. Levi, A. Mura, A. Godone, "Planar-waveguide external cavity laser stabilization for an optical link with 10-19 frequency stability," IEEE Trans. Ultrason. Ferroel. Freq. Contr., vol. 58, pp. 2582-2587, Dec. 2011.

[8] G. Bolognini, S. Faralli, A. Chiuchiarelli, F. Falconi and F. Di Pasquale, "High-power and low-RIN lasers for advancedfirst- and higher-order Raman co-pumping," IEEE Photonics Technol. Lett., vol.18, pp. 15911593, Aug. 2006.

[9] C. R. S. Fludger, V. Handerek, and R. J. Mears, "Pump to Signal RIN Transfer in Raman Fiber Amplifiers," IEEE J. Lightwave Technol., vol. 19, pp. 1140-1148, Aug. 2001.

[10] W. Williams, W. C. Swann, and N. R. Newbury, "High-stability transfer of an optical frequency over long fiber optic links," J. Opt. Soc. Am. B, vol. 25, pp. 1284-1293, Aug. 2008.

[11] S. T. Dawkins, J. J. McFerran, A. N. Luiten "Considerations on the Measurement of the Stability of Oscillators with Frequency Counters," IEEE Trans. Ultrason. Ferroelectr. Freq. Control, vol. 54, pp. 918-925, May 2007.

[12] F. Levi, R. Ambrosini, D. Calonico, C. E. Calosso, C. Clivati, G. A. Costanzo, et al., "LIFT: The Italian Fiber Network For Frequency and Time Distribution", this proceedings. 